\title{
RESYSTER: A HYBRID RECOMMENDER SYSTEM FOR SCRUM TEAM ROLES BASED ON FUZZY AND ROUGH SETS
}

\author{
RICARDO COLOMO-PALACIOS, ISRAEL GONZÁLEZ-CARRASCO, \\ JOSÉ LUIS LÓPEZ-CUADRADO, ÁNGEL GARCÍA-CRESPO \\ Department of Computer Science \\ Carlos III University, Av. Universidad 30-28911, Leganes (Madrid), Spain \\ e-mail: \{ricardo.colomo, israel.gonzalez\}@uc3m.es \\ \{joseluis.lopez.cuadrado, angel.garcia\} @uc3m.es
}

\begin{abstract}
Agile development is a crucial issue within software engineering because one of the goals of any project leader is to increase the speed and flexibility in the development of new commercial products. In this sense, project managers must find the best resource configuration for each of the work packages necessary for the management of software development processes in order to keep the team motivated and committed to the project and to improve productivity and quality. This paper presents ReSySTER, a hybrid recommender system based on fuzzy logic, rough set theory and semantic technologies, aimed at helping project leaders to manage software development projects. The proposed system provides a powerful tool for project managers supporting the development process in Scrum environments and helping to form the most suitable team for different work packages. The system has been evaluated in a real scenario of development with the Scrum framework obtaining promising results.
\end{abstract}

Keywords: fuzzy set, rough set, Scrum, work package, recommender system.

\section{Introduction}

Software systems are designed and coded by humans. It can be said that software engineering work is highly dependent on people (Colomo-Palacios et al., 2011) and rich in human capital (Colomo-Palacios et al., 2010). In other words, software development is a human centric and sociotechnical activity (Casado-Lumbreras et al., 2011) influenced by personnel factors.

Given that software workers do not work in isolation, according to Moe et al. (2010), software development depends significantly on team performance, as does any process that involves human interaction. Team composition is one of the main issues in project management; it has firsthand effects on creating organizational performance, so it becomes a very critical issue in today's rapidly changing company environment (Wi et al., 2009). As a result, software development team composition issues are one of the principal challenges of software project management due to their sheer complexity (Chang et al., 2001) as it dramatically influences project performance (Chi and Chen, 2009). A failure in the formation of project teams can manifest itself as a decrease in service quality, unattainable project deadlines (with expected financial penalties), and loss of credibility (Strnad and Guid, 2010).

Properly assigning people to development roles is crucial for creating productive software development teams (Acuña and Juristo, 2004). In contrast, wrong assignments may result in a significant loss of value due to under-staffing, under or over-qualification of the assigned personnel, and high turnover of poorly matched workers (Naveh et al., 2007). In this complex scenario, software managers typically assign people to project teams according to their experience, heuristic knowledge, subjective perception, and instinct (Acuña et al., 2006). Performing this task without the help of proper computer-enabled tools is tedious, repetitive and time-consuming (Naveh et al., 2007).

On the other hand, agile methods have been gaining acceptance in the mainstream software development community (Lee and Yong, 2010). Agile development approaches differ from the traditional, plan-driven, structured approaches as the former put more emphasis on lean processes and dynamic adaptation than on detailed front-end plans and heavy documentation (Nerur 
and Balijepally, 2007). Many efforts embraced the new philosophy coming from the Agile Manifesto. These approaches include eXtreme Programming (XP) (Beck, 2001), Scrum (Schwaber and Beedle, 2002), Feature Driven Development (FDD) (Palmer and Felsing, 2002), Crystal (Cockburn, 2000) and Adaptive Software Development (ASD) (Highsmith and Orr, 2000), to cite some of the most relevant ones. Focusing on Scrum, according to a recent report issued by West et al. (2010), this effort makes agile adoption the most adopted agile methodology. Regarding team composition, Scrum postulates self-organizing team structures and flexible collaborations in a flat hierarchy. Thus, the building of a team in this setup may be particularly challenging, given the inclusive yet fluid nature of teams in this context (Licorish et al., 2009).

Our prior research on recommender systems, in heterogeneous domains, have demonstrated the validity of the integration of various intelligent components in order to obtain a viable platform from a technical perspective. Sem-Fit (Garcia-Crespo et al., 2011a) is a semantic hotel recommendation expert system, based on the consumer's experience about recommendation provided by the system. Sem-Fit uses the consumer's experience point of view in order to apply fuzzy logic techniques to relating customer and hotel characteristics, represented by means of domain ontologies and affect grids. Meanwhile, SINVLIO (Garcia-Crespo et al., 2011b) is a tool based on semantic technologies and fuzzy logic techniques that recommends investments grounded in both psychological aspects of the investor and traditional financial parameters of the investments. Finally, SEMO (Garcia-Crespo et al., 2010 ) is a platform for customer social networks analysis based on semantics and emotion mining. The platform benefits from both semantic annotation and classification and text analysis, relying on techniques from the natural language processing domain.

In this scenario, the aim of this paper is to present ReSySTER, a hybrid recommender system for Scrum team roles based on fuzzy logic, rough set theory and semantic technologies. Following the path drawn by previous efforts devoted to employ computational intelligent techniques to support the formation of teams, ReSySTER gives recommendations for team formation in Scrum driven projects based on hybrid techniques. With regard to new aspects tackled in this paper, ReSySTER is an evolution of previous works that has been tested in a new environment. This new environment is a software engineering scenario or, more precisely, Scrum. Regarding the evolution of the architecture, our previous works showed various applications of recommender systems based on semantics and fuzzy logic. In this work, we integrate rough sets with this proven architecture.

\section{Literature review}

According to Salo and Abrahamsson (2008), Scrum is aimed at providing an agile approach to managing software projects while increasing the probability of a successful development of software. Emphasizing that small cross-functional teams produce better results, Scrum was first described as "the rugby approach" by Takeuchi and Nonaka (1986). Central to Scrum is the idea that many of the processes during the development cannot be predicted and therefore it addresses the software development in a flexible way (Vlaanderen et al., 2011). There are just two stages that are fully defined: the planning and the closure. In between, the final product is developed by several teams in a series of flexible black boxes called 'sprints'. This flexibility is described by Pino et al. (2010): "Scrum is not a process or a technique for building products; rather, it is a framework within which you can employ various processes and techniques".

With regard to the people working in Scrum projects, self-management is a defining characteristic in Scrum (Moe et al., 2010). The team is accorded full authority to do whatever it decides is necessary to achieve the goal (Schwaber and Beedle, 2002). Thus, a Scrum team is given significant authority and responsibility for many aspects of their work, such as planning, scheduling, assigning tasks to members, and making decisions (Moe et al., 2010).

Three roles, the product owner, team, and Scrum master, make up all together the Scrum team (Schwaber and Beedle, 2002). The product owner is the one person responsible for a project's success. He or she is responsible for maximizing return on investment and bears profit and loss responsibility for the product. The Scrum team is a collective role that must be played by all the team members (Fuentes-Fernandez et al., 2010). This cross-functional team builds the product including tasks like analysis, design, construction and test. The Scrum team is typically made up of 5-9 people. Finally, the Scrum master is the coach and main decision-maker in the development team. He or she makes sure that every team member follows and intends Scrum practices.

Although there are several distributed Scrum practices reported in the literature and tested in the industry (e.g., Lee and Yong, 2010), the inner complexity of standard Scrum team building is notable (Licorish et al., 2009).

The application of intelligent and soft-computing techniques to personnel selection issues has a long tail in the literature. Confronting with Malinowski et al. (2008), there are many efforts devoted to selecting individuals supported by information systems and by computational intelligence methods (e.g., Celik et al., 2009; Toroslu and Arslanoglu, 2007; Strnad and Guid, 2010). On the software development projects arena, the work of 
Mohanty et al. (2010) contains a recent and relevant review on the topic. More precisely, the efforts to use these technologies in personnel issues are frequent (e.g., Barreto et al., 2008; Barcus and Montibeller, 2008). Focusing on the technologies employed in ReSySTER, namely, rough sets, fuzzy techniques and semantic technologies, these efforts are also present in the software engineering staffing literature: rough sets (Imai et al., 2008)), fuzzy techniques (Strnad and Guid, 2010; Wang and Lin, 2003) and semantic technologies (Garcia-Crespo et al., 2009; Valencia-Garcia et al., 2010). The combination of methods for improving the performance of intelligent systems is a common issue addressed by multiple authors in multiple domains ( $\mathrm{Li}$ and Chiang, 2011; Nowicki, 2010; Mahmoud, 2011; Zhong and Skowron, 2001). However, to the best of the authors' knowledge, there is no effort devoted to apply these computational intelligence techniques into the formation of Scrum teams.

The International Project Management Association (IPMA 1 ) is a federation of national project management associations. The IPMA's four-level certification program takes into account the most relevant competences for project managers and project teams. The documentation developed by the IPMA and their partners identify the main competences involved in project management and project teams. Each country has a version of these competences: this paper considers the USA National Competence Baseline (USANCB) 2 . This definition allows sharing vocabulary in the definition of competences as well as establishes a measure of the competence level of each Scrum role.

\section{ReSySTER: A hybrid recommender system for Scrum team roles}

This section describes the architecture of ReSySTER, designed to assist project managers in configuring the best team for the work packages present in projects managed under Scrum. This recommendation is based on determining the best team based on the SAP and the competences required for each work package which is the best team taking into account the resources available.

The main steps in the recommendation process within the ReSySTER framework are displayed in Fig. 1 and could be summarized in the following topics:

1. Labeling of competences: Each WP presents a set of competencies needs coded with respect of the USA NCB standard (Benjamin et al., 2008). The labeling of these competences within the three major groups defined by USANCB, contextual, technical and behavioral, will be conducted by the semantic

${ }^{1}$ http://www.ipma.ch

${ }^{2}$ http: / / www. asapm.org/resources/USA_NCB.pdf component of this framework. For each competence the project manager can set the numerical level (0-100) that should reach the personnel assigned to this WP (known as the competence level). Furthermore, the project manager could set the team size requested for each WP. In addition, the SAP set is defined by a personal profile and a set of professional competences. The project manager is responsible for labeling the different features of each member of the staff following the guidelines of the ontology defined in the semantic component of the framework.

2. Fuzzy transformation: Inside ReSySTER, the numerical values (competence level) of the WP competences will be transformed into a linguistic value based on the IPMA level certification (Caupin et al., 2006). For this purpose, a fuzzification process is achieved based on the fuzzy sets and fuzzy rules defined within the fuzzy component of the framework. Furthermore, the personal profile and the professional competences of the staff with continuous values are transformed into discrete values through a fuzzification process based on fuzzy sets. Through this process the potential uncertainty associated with these variables is reduced and the matching between the staff competences and the competences required by the WPs is facilitated.

3. Rough set categorization: To facilitate ReSySTER recommendations, firstly it is necessary to know the level of competence of each person of the SAP within the contextual, technical and behavioral competences of the WP. To establish the rules that automatically transform the level of competence from personal characteristics and professional competences to the USANCB standard, ReSySTER includes a rough set approach based on a set of earlier assessments. The rules obtained are incorporated in the system to estimate the different levels of SAP competences based on the IPMA levels required by the WP.

4. Matching and recommendation process: For each WP the project manager can establish different competences required as well as the order of importance within the project scope. To facilitate this process, a component of user interaction based on fuzzy logic has been included: the Work Package Matrix (WPM). With this matrix, the project manager can set for each competence of the WP different levels of importance, by means of associating a linguistic label to establish their priorities in a logical order intelligible to humans.

Once the different WPs of the project are defined (with the level of competence for the contextual, technical and behavioral elements), the project 


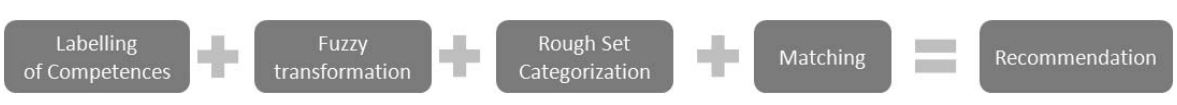

Fig. 1. Steps in the ReSySTER recommendation process.

manager selects one WP and chooses a set of candidates from the SAP (but there is also available a recommendation based on the whole staff). Then, the personal profile and the professional competences of the candidates are transformed into USANCB competences by means of the fuzzification process and rough set rules. As a result of these processes the Human Resource Matrix (HRM) is created for each WP.

Afterwards, ReSySTER evaluates each candidate of the HRM and then begins the matching process between the candidate and the competences defined in the WPM for this WP. For this purpose, during the defuzzification process the system applies the fuzzy rules obtaining a value between " 0 " and " 1 " for each matching between the matrices. A zero value is the lowest level of adequacy for the work package and the value " 1 " is the highest level of adequacy. When a matching is found, the value " 1 " is weighted by ReSySTER according to the associated linguistic label.

Finally, ReSySTER recommends the most suitable team for this WP based on different features (best team, minor gap, ranked teams, etc.). In some situations several iterations of the matching process will be required. When more than one WP takes part in the recommendation process, ReSySTER will iterate in order to solve possible conflicts that would appear in the matching between the SAP and WP sets. Moreover, two types of user has been identified: project managers and experts. The project manager in ReSySTER is the person in charge of configuring the recommendations (defining the size and the features of the team), and the expert is the person in charge of tuning the system (adjusting the fuzzy sets, fuzzy rules and rough sets).

Therefore, the main components of this framework are semantic and ontology labeling, categorization based on rough sets theory, and decisions based on fuzzy sets and fuzzy rules. In Fig. 2 these components within the ReSySTER framework are exposed. They will be fully detailed in the next sections.

3.1. Semantic and ontology description. For categorizing both WP and SAP sets, a number of competences have been selected. On the one hand, competences are related to the professional aspects required for the WP, and to the characteristics of the people that can work on each WP. Once the WP and SAP characteristics had been defined, they were formalized in the form of an ontology. An ontology is the formal representation of a shared conceptualization (Gruber, 1993). In this way, the terms, relationships and rules are formally described in a defined language (in this case, OWL-DL 3 ). The aim of the developed ontology is to provide a closed domain of competences and characteristics that ease the characterization of WP requirements and SAP profiles.

Figure 3 represents WP competences obtained from the list of the IPMA and the USANCB (Caupin et al., 2006) which establishes a set of competences required for projects. These competences are classified into three groups: contextual competences, related to management, technical competences and behavioral competences. Work packages will be characterized by these competences with a level associated. Each WP competence is rated from 0 to 100 according to the required competence level. The approximation of this value is subjective and is provided by the project manager according his/her knowledge about the project and the concrete characteristics of the WP.

The SAP of the company has been also characterized in order to allow the matching between the available SAP and the requirements of the WP. The characterization of the SAP using the same parameters as the WP would require the evaluation of each one according the certification rules. Due to the inherent difficulties of this process, the personal aspects of the SAP are represented by more general characteristics that will be used later for the evaluation of the competency level of the SAP.

Figure 4 depicts the ontology that represents the characteristics of the SAP. These characteristics are classified into two different groups. On the one hand, personal characteristics of the SAP are represented in order to represent relevant aspects of SAP indirectly related to the professional aspects. Age, gender or incomes are included in this group. On the other hand, the second group represent characteristics related to the professional competences required for WPs. These competences are also characterized by a value from 0 to 100 according to the required competence level. The knowledge relative to these characteristics is required for determining the SAP competency level for each WP competency. The values for each characteristic is obtained from the professional profile of each SAP.

$\sqrt[3]{\text { http: / / www.w3 org/TR/owl-features/ }}$ 


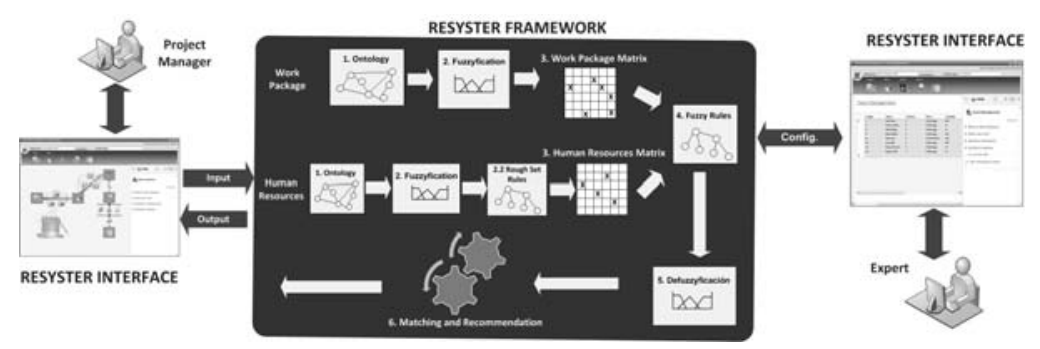

Fig. 2. ReSySTER interface and framework.

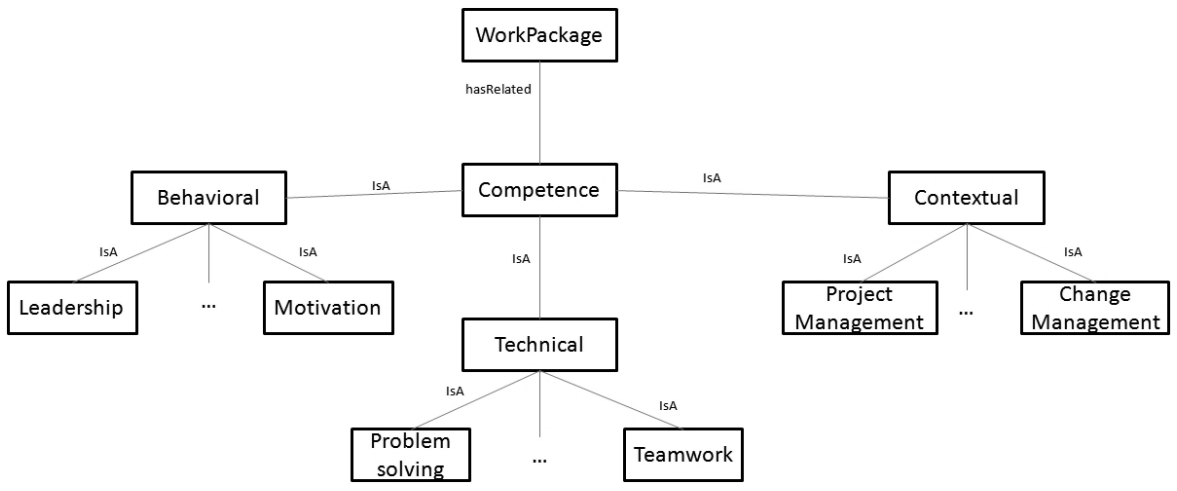

Fig. 3. ReSySTER ontology: WP competences (partial view 1).

3.2. Fuzzy transformation and fuzzy sets components. After the labeling of the SAP and WP subsets with the semantic component, the fuzzy transformation takes place. Briefly, fuzzy sets theory provides a framework for the representation of the uncertainty of many aspects of human knowledge. Nowadays, fuzzy rule based systems are successfully applied to a wide range of real-world problems from different areas (Hadavandi et al., 2010) and in many real-world applications (Do et al., 2010; Li and Chan, 2011; Qin et al., 2011). Although a system can be defined mathematically in a general sense, a fuzzy logic system representation is still preferred by engineers (Liu et al., 2010).

In the first place, ReSySTER will characterize the members of the staff available for the project according to their personal characteristics exposed in Section 3.1. This variables forms the SAP cluster and some of the variables of this cluster could be characterized by fuzzy values, which would lead to a fuzzy set for each of the variables. In this context, the variables affected by the fuzzification process are grouped in the $C_{S A P}$ fuzzy cluster and they are enumerated in the following set:

$$
\begin{aligned}
& C_{S A P} \\
& =\{\text { Age, Salary, YearsIn Company, Experience }\} .
\end{aligned}
$$

Secondly, ReSySTER will transform the numerical value (known as competence levels) associated with each variable of the WP cluster into a discrete value through the fuzzyfication process. The WP cluster is split in three subsets (contextual, technical and behavioral competences), and each one is characterized with several variables. In this case, all the variables of these subsets will be affected by the fuzzyfication process resulting in the fuzzy sets $F_{C o n}, F_{T e c}, F_{B e h}$. All these fuzzy sets are grouped in the $C_{W P}$ fuzzy cluster and enumerated in Eqn.1.

$$
C_{W P}=\left\{F_{C o n} A N D F_{T e c} A N D F_{B e h}\right\} .
$$

Furthermore, in the design and implementation of a fuzzy logic system, the designer has to decide which of the three most popular membership functions will be used: triangular, Gaussian or trapezoidal (Negnevitsky, 2005). In this paper, the triangular and trapezoidal membership functions have been used for the fuzzy sets. Using triangular and trapezoidal functions means that the performance rate will be very fast, although the level of accuracy will be lower than with either of the membership functions (Xie et al., 1998).

The triangular function is defined by a lower limit $a$, an upper limit $b$, and a value $m$, where $a<m<b$

$$
\mu_{A}(x)=\left\{\begin{array}{lll}
0 & \text { if } \quad x<a, \\
\frac{x-a}{m-a} & \text { if } \quad a<x \leq m, \\
\frac{b-x}{b-m} & \text { if } \quad m<x<b, \\
0 & \text { if } \quad x \geq b .
\end{array}\right.
$$




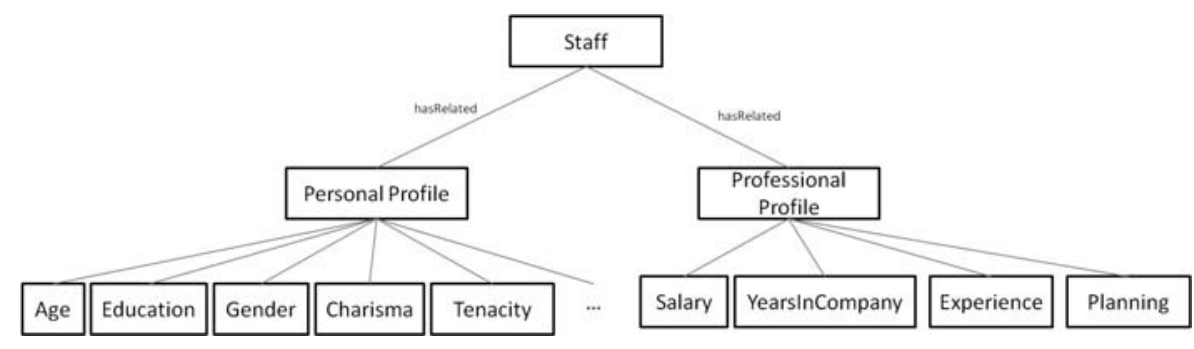

Fig. 4. ReSySTER ontology: SAP characteristics (partial view 2).

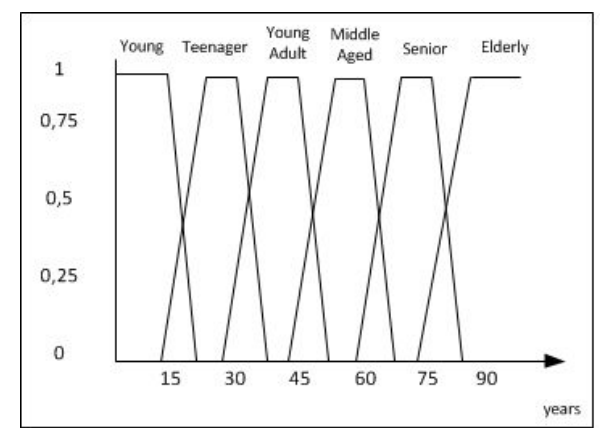

Age Membership function

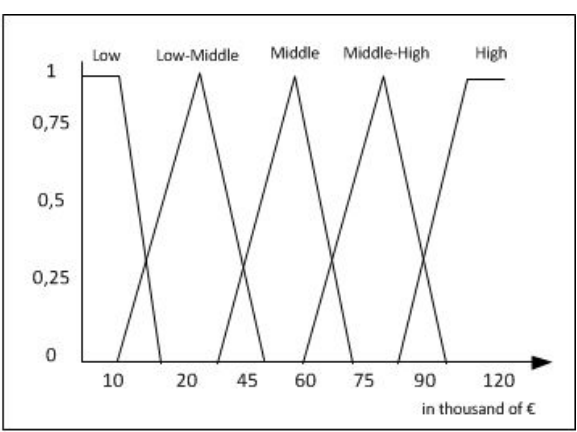

Salary Membership function

Fig. 5. Membership function for the Age and Salary sets.

The trapezoidal function is defined by a lower limit $a$, an upper limit $d$, a lower support limit $b$, and an upper support limit $c$, where $a<b<c<d$,

$$
\mu_{A}(x)=\left\{\begin{array}{lll}
0 & \text { if } \quad x<a \text { or } x>d, \\
\frac{x-a}{b-a} & \text { if } \quad a \leq x \leq b, \\
1 & \text { if } \quad b \leq x \leq c, \\
\frac{d-x}{d-c} & \text { if } \quad c \leq x \leq d .
\end{array}\right.
$$

There are two special cases of a trapezoidal function, which are called R-functions and L-functions. In the R-former the parameters $a=b=-\infty$,

$$
\mu_{A}(x)=\left\{\begin{array}{lll}
0 & \text { if } \quad x>d \\
\frac{d-x}{d-c} & \text { if } \quad c \leq x \leq d \\
1 & \text { if } \quad x<c
\end{array}\right.
$$

In the L-later the parameters $c=d=+\infty$,

$$
\mu_{A}(x)=\left\{\begin{array}{lll}
0 & \text { if } \quad x<a, \\
\frac{x-a}{b-a} & \text { if } \quad a \leq x \leq b, \\
1 & \text { if } \quad x>b .
\end{array}\right.
$$

The traditional notation for fuzzy sets was defined by Zadeh (1965) and it has been used in ReSySTER for the definition of SAP and WP fuzzy sets. After defining these fuzzy sets, the fuzzy inference rules may be used to represent the relation between the fuzzy sets.

Focusing on the SAP cluster, the fuzzy sets will transform the continuous values of some variables into discrete values. The fuzzy membership has been been defined taking into account the knowledge of the experts holding interviews with them. In Figs. 5 and 6 the memberships functions of fuzzy sets within the SAP cluster are exposed.

In the case of the WP, the fuzzy sets will determine the IPMA level associated with each one, taking into account the competence level of each competence (contextual, technical and behavioral variables) defined by the project manager. The IPMA level is defined with four categories: A, B, C and D (Caupin et al., 2006). The fuzzy set applied to transform the numerical value of the competence level to a discrete value based on IPMA categories is depicted in Fig. 7

Finally, the main goal of this process is to reduce the potential uncertainty associated with the continuous variables and to facilitate for the project manager the matching between the staff competences and the competences required by the WPs.

3.3. Rough sets. Rough logic and rough sets theory are mainly devoted to analysis of incomplete, uncertain, and inconsistent data (Kluska-Nawarecka et al., 2011). 


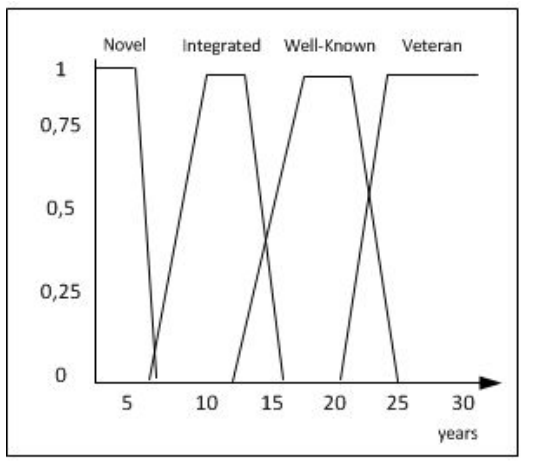

Years in Company

Membership function

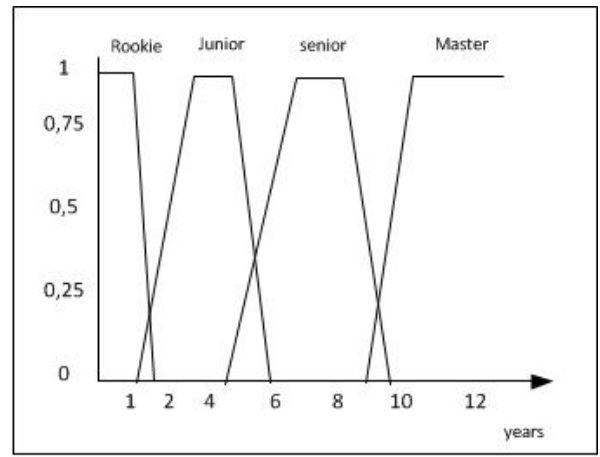

Experience

Membership function

Fig. 6. Membership function for the Years in Company and Experience sets.

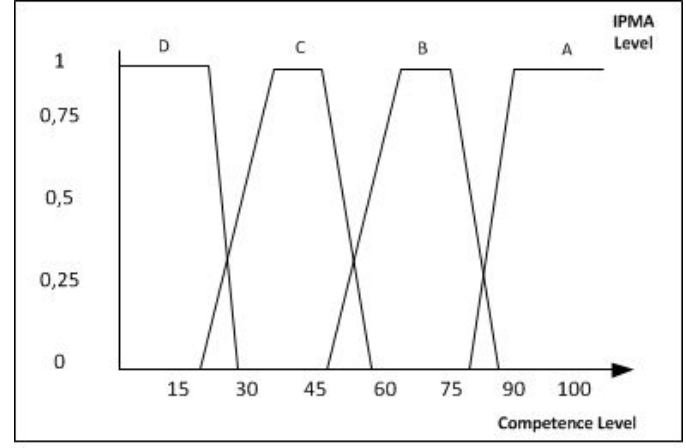

Example of Membership function for WP Competence

Fig. 7. Example of a membership function for WP competences.

The rough set approach was proposed initially by Pawlak (1982). It is based on the classification of elements into sets according to equivalence classes. According to these classes, three regions are defined (see Fig. 8). The upper approximation determines the set of elements which can be unambiguously classified as part of the target set. Finally, the boundary region is the set of elements which cannot be definitely classified as part of the target set. From these premises, each element of the information system is characterized by two types of attributes: condition attributes, which characterize the elements, and decision attributes, which determines the equivalence classes. Part of the process implies the reduction of attributes in order to obtain the set of more relevant attributes for the decision. As a result, a number of decision rules are extracted which can be used in decision processes. Finally, just like the other intelligent components of ReSySTER, rough sets systems have been successfully applied to a wide range of real-world problems from different areas (Kluska-Nawarecka et al., 2011; Malyszko and Stepaniuk, 2011).

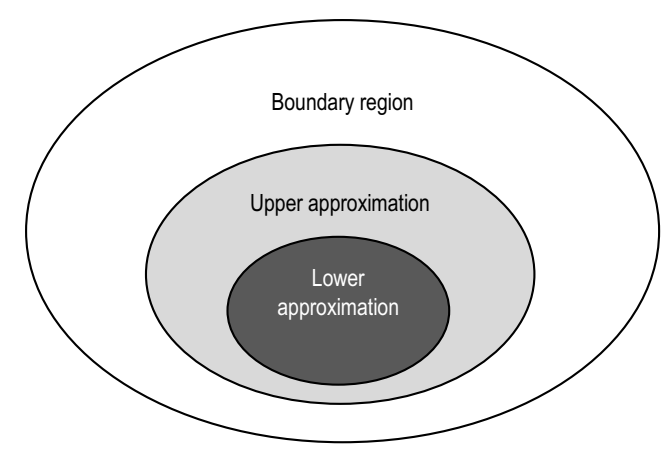

Fig. 8. Regions in rough set theory.

In this research, rough set theory was applied for the identification of rules for classifying the SAP set in the different levels of each competence. Since the SAP set has been characterized using more general characteristics, the proposed approach was useful for finding the relationships between the personal and professional characteristics of the SAP and the competency level. Thus, each competency level of each competency was represented using this approach, obtaining on the one hand the set of relevant attributes for approaching the competency level of a competency and on the other the rules for this classification, as shown in Table 1.

First, a set was defined for each competency to be evaluated. As mentioned, the decision attribute is the competency level and the rest of attributes has been represented in the form of an ontology, as shown in Section 3.1. Secondly, the number of condition attributes was reduced for each competency. Finally, the set of decision rules was extracted in order to be applied in the classification of new SAP sets.

3.4. Matching and recommendation. The last steps of the ReSySTER framework are the matching and the 
Table 1. Information table (partial view).

\begin{tabular}{|c|l|c|l|l|}
\hline SAP & $\begin{array}{l}\text { Years in } \\
\text { Company }\end{array}$ & Experience & $\cdots$ & $\begin{array}{l}\text { Comp. } \\
\text { Level }\end{array}$ \\
\hline \hline 1 & Veteran & Master & $\ldots$ & A \\
2 & Novel & Senior & $\ldots$ & B \\
3 & Integrated & Junior & $\ldots$ & C \\
4 & Integrated & Senior & $\ldots$ & B \\
5 & Novel & Roookie & $\ldots$ & D \\
$\vdots$ & $\vdots$ & $\vdots$ & $\vdots$ & $\vdots$ \\
\hline
\end{tabular}

Table 2. Example of WPM builds for WP1.

\begin{tabular}{|c|c|c|c|}
\hline WP1 & Comp. 1 & Comp. 2 & Comp. 3 \\
\hline \hline Level A & Essential & - & - \\
Level B & Desirable & Desirable & - \\
Level C & - & Essential & \\
Level D & Not Desirable & - & \\
\hline
\end{tabular}

recommendation process. The former is divided in three different stages: building of the WPM and HRM matrices, application of fuzzy rules and defuzzification of the internal values back to the real world in order to obtain a quantifiable result. These stages are depicted in Fig. 2 as Steps 3-5 of the framework.

3.4.1. WPM and HRM matrices. In the case of the WP, after the definition of the competence level and the creation of the fuzzy sets, the project manager has for each WP a set of competences labeled with IPMA levels. Thus, in this step, the project manager builds the WPM matrix specifying, for each competence and the IPMA level, the priority level required. This Priority Level (PL) is labeled with a linguistic label and it could be a positive or negative indicator. The set of priority levels defined in ReSySTER, in the order of importance, is the following:

$$
\begin{array}{r}
P L_{W P}=\{\text { Essential, Desirable, Indifferent, } \\
\text { NotDesirable, Never }\} .
\end{array}
$$

In Table 2 an example of this matching between the competences and IPMA level for one WP is shown. The columns tagged in this table with "Comp." refer to the set of competences.

In the case of the SAP, in the previous step of the framework the staff members have been labeled for each competence with an IPMA level through the rough set component. This component enables the tagging of staff members including the rules obtained from the rough sets component to determine for each competence the associated IPMA level.

As a result of this component, ReSySTER builds the HWM matrix specifying for each member of the SAP subset the IMPA level associated with each competence.
Table 3. Example of HRM builds for the staff member.

\begin{tabular}{|c|c|c|c|}
\hline SAP & Comp. 1 & Comp. 2 & Comp. 3 \\
\hline \hline John Doe & Level C & Level C & Level D \\
Peter Smith & Level A & - & Level A \\
Joe Blogg & - & Level B & - \\
Mary Major & - & Level C & Level D \\
Jane Roe & Level C & Level C & Level B \\
\hline
\end{tabular}

In Table 3 an example of this matching between Competences and IPMA level for the members of the SAP subset is shown. The columns tagged in this table with "Comp." refers to the set of competences.

3.4.2. Fuzzy rules. Afterwards, the following step make by ReSySTER is to transform the WPM matrix in fuzzy rules. These fuzzy rules will have the following format: "If premise(s) Then conclusion". The premises zone includes logic links referring to the IPMA level of a competence, whereas a conclusion is not more than the deduction of a fact from the premises of the rule. Thus, taking into account the example of WPM depicted in Table 2, the following rules will be created for Competence 1:

$$
\begin{aligned}
& R_{C o m p 1}=\{ \\
& R_{1}=\text { IF Level }(I P M A)=A \text { THEN } \\
& \text { Comp1 IS Essential } \\
& R_{1}=\text { IF Level }(I P M A)=B \text { THEN } \\
& \text { Comp1 IS Desirable } \\
& R_{1}=\text { IF Level }(I P M A)=D \text { THEN } \\
& \text { Comp1 IS Not Desirable } \\
& \}
\end{aligned}
$$

Furthermore, the competences fuzzy rules $\left(R_{C o m p}\right)$ associated with a specific WP could be merged in a more complex rule $\left(R_{W P}\right)$. The complex rule enables the selection of the best candidate of the staff. For this purpose the premises of the rules are all the competences $\left(C_{i}\right)$ involved with the corresponding priority level. When the rule is triggered by ReSySTER, the conclusion of the rule determine the calculation of the value associated to this candidate of the staff $\left(P_{\text {member }}\right)$.

Taking into account the WPM of Table 3 , an example of a complex rule is

$$
\begin{aligned}
& R_{W P}=\{ \\
& \text { IF } P L_{C 1}\left(\text { Level }_{A}\right)=\text { Essential AND } \\
& P L_{C 2}\left(\text { Level }_{B}\right) \text { AND } P L_{C 3}\left(\text { Level }_{C}\right) \\
& \text { THEN Calculate }\left(P_{\text {member }}\right)
\end{aligned}
$$


Therefore, to be able to obtain the full set of fuzzy rules that model all the WPs of the current project, all theoretically possible combinations of $R_{t}$ rules were considered, taking into account the number of antecedents $p$ and the number of input fuzzy sets $A_{p}$ considered for each antecedent. Thus, for each consequent, the theoretical number of possible rules is expressed as

$$
R_{t}=\prod_{n} A_{n} \quad \text { for } \quad n=1, \ldots, p .
$$

3.4.3. Defuzzification. The last step is the defuzzification process, which corresponds with the conversion of the linguistic values to numerical values. This process is carried out thanks to the fact that the labels of $P L_{W P}$ have a weight or numeric value associated. An expert is in charge of fine tuning of this values.

Lastly, taking into account the HRM and WPM matrices as well as the fuzzy rules $R_{t}$, ReSySTER calculates the score that is achieved by each of the staff members depending on the rules triggered and the associated weights. Each member of the staff obtains therefore a total score that depends directly on its personal and professional profile and its relevance to a specific WP.

As has been formerly commented, there are different parameters which allow configuring the tool in order to obtain more refined results. The expert has at his/her disposal in the first place two general parameters which allow delimiting and optimising the results obtained by the tool.

Finally, the expert can use in ReSySTER two parameters to adjust the calculation process and to improve the obtained recommendations. The first parameter available is the optimization degree. It refers to the algorithm used to calculate the total score that a member of the SAP achieves during the searching process. The three algorithms implemented are basic, sensitive and normal. The first one, basic optimization, marks as valid all of the members that fulfill some competence of the WP; in other words, some of the rules are activated during the recommendation process. The second option, sensitive optimization, multiplies the weight of all the rules that are triggered $\left(R_{i}\right)$ for this WP and member of the staff. Thus, the total score $\left(P_{\text {member }}\right)$ is calculated following Eqn. (7)

$$
P_{\text {member }}=\prod\left(P L_{R_{i}}\right)
$$

where $P_{\text {member }}$ is the total score achieved by the evaluated member of the staff depending on the multiplication of the numeric values of $P L$ set for the activated rules $R_{i}$.

The third option, normal optimization, sums the PL values of all the rules that are triggered $\left(R_{i}\right)$. Hence, the total score of each of the products is calculated following

$$
P_{\text {member }}=\sum\left(P L_{R_{i}}\right)
$$

The process of recommendation in ReSySTER is based firstly on the activation of the rules defined in the fuzzy process for each member of the SAP. Secondly, and from the mathematical point of view, partial scores are calculated for each of the rules that are triggered for that member. Finally, ReSySTER implements three different variants to calculate the accumulative score at the end of the recommendation process for all the rules activated: normal (sum of the partial scores of each rule), sensitive (product of the partial scores of each rule) and basic (activation of any rule).

The last mode, the basic algorithm, allows locating those members of the SAP that accomplish some of the rules created by the expert. In this case, ReSySTER does not calculate aggregate scores because it only considers those members of the SAP that satisfy some of the rules, and thus returns them as possible candidates.

Therefore, depending on the type of algorithm selected by the expert, for a particular query ReSySTER can tune the final scores obtained by each of the members of the SAP. Thus, the expert can change the behavior of ReSySTER, according to the rules created for the matching and recommendation process, to obtain different sets of recommendations. The aim of implementing different scoring algorithms (normal, sensitive and basic) is to be able to adapt to changing environments where the expert cannot know in advance what scoring process is most appropriate.

The second parameter to be configured by the project manager is the size of the team for each WP. Hence, this parameter allows delimiting the number of members of the staff to be shown to the project manager after the searching process. Equation (9) determines the size of the team, specified with $n$ in the case of its being zero, all the members evaluated would be returned ordered by score,

$$
P_{\text {member }}=\max _{n}\left(\left[P_{\text {member }}\right]\right)
$$

This turns out to be very useful when the available SAP set is very large and the members of the staff have very similar profiles as the system would not be able to differentiate between them because they would obtain a very similar score. Furthermore, the expert can modify the numeric values of the PL set and also the textual descriptions associated with these values.

In short, ReSySTER allows using different optimization degrees while the expert can change dynamically the results that are offered to the project manager depending on the necessities of the WP and the availability of the SAP in each moment.

3.5. Operation example. For this example we have five candidates for a given work package. Firstly, their characteristics are represented by means of the SAP ontology (Fig. 4). In this example we select "Years in 
Table 4. Numerical values for the sample characteristics.

\begin{tabular}{|c|c|c|}
\hline SAP & Years in Company & Experience \\
\hline \hline John Doe & 6 & 8 \\
Peter Smith & 0 & 0 \\
Joe Blogg & 2 & 3 \\
Mary Major & 7 & 7 \\
Jane Roe & 6 & 6 \\
$\vdots$ & $\vdots$ & $\vdots$ \\
\hline
\end{tabular}

Table 5. Sample of fuzzification and level determination.

\begin{tabular}{|c|l|c|l|l|}
\hline SAP & $\begin{array}{l}\text { Years in } \\
\text { Company }\end{array}$ & Experience & $\cdots$ & $\begin{array}{l}\text { Comp. } \\
\text { Level }\end{array}$ \\
\hline \hline John Doe & Integrated & Senior & & B \\
Peter Smith & Novel & Roookie & & D \\
Joe Blogg & Novel & Junior & & C \\
Mary Major & Integrated & Senior & & B \\
Jane Roe & Integrated & Senior & & B \\
$\vdots$ & $\vdots$ & $\vdots$ & $\vdots$ & $\vdots$ \\
& & & & \\
\hline
\end{tabular}

Company" and "Experience". The particular values for each characteristic are shown in Table 4

After the characterization of the candidates, their characteristics are fuzzified, according the fuzzy sets, and their membership functions defined with the help of the experts (Fig. 6). Previously, based on a set of examples and the expert criteria, the rough sets were defined and the rules of pertinence to these sets were established. In this way, the rules for approaching the competence level based on the personal and professional characteristics of each candidate were determined. The last column of Table 5 shows the competence level for each candidate for the "Project Management" competency.

This process is repeated for each of the competences required for the work package WP1 (defined in Table 2). The work package is also characterized by selecting the competencies required and the level required for each one, as shown in Table 2 After these steps the matching process is executed and the fuzzy rules are applied to the candidates in order to determine the suitability of each candidate for the WP. Finally the deffuzification process translates the fuzzy values of each candidate into a score for each candidate. The candidates with the highest scores will be offered. The number of candidates suggested by the system is determined by the size of the team (see Eqn. (9)).

\section{Evaluation}

4.1. Research design. With the aim of obtaining results and feedback about the work performed, an evaluation was carried out. This evaluation checked all the different aspects concerning all features of ReSySTER.
In order to do so, real competence information about people involved in Scrum projects was necessary. The subjects adopted the project manager role in these software projects as defined in Section 2 and they were selected from those who answered positively to a personal invitation. This invitation was sent by the authors among personal contacts working in Scrum projects in leading roles for more than two years.

The tests performed for the evaluation were divided into two phases:

1. Phase 1. This phase is divided into two different tests. The aim of these tests was to obtain only one team for a specific WP under different situations. Hence, this team could be achieved using two versions:

(a) Search for the best general team for a specific WP. In this case the best team is selected regardless of the competences defined for the WP.

(b) Search for the team with a minor gap for a specific WP. This test searches for the team that fits most the competences desired for a WP.

2. Phase 2. In this phase several WPs were taken into account. The aim of this test was to obtain ranked teams. Therefore, all the teams must be evaluated for all WPs in order to assure the best fit for all them.

The configuration of ReSySTER for these tests was the following:

- Phase 1: Team composed of 4 members and the optimization degree sensitive in Test 1 and normal in Test 2.

- Phase 2: Team composed of 3 members and the optimization degree was sensitive.

For the evaluation process, 10 real WPs were defined by the project manager according to their USANCB competences following the stages of the framework defined in Section 3 Next, in Phase 1, only one WP was selected to perform Test 1 and Test 2. In Phase 2, all the WPs were selected and all of them were involved in the recommendation process. In all the cases, to achieve the evaluation of ReSySTER, each expert gives two possible teams for each WP. Agreement among the experts for the recommendations was achieved using the Delphi method. Simultaneously, ReSySTER obtains a set of two recommendations for each WP using the matching process described in Section 3.4

For evaluating the performance of the working team selection process, Precision and Recall and F1 measures were used to measure the degree of relevance of the recommendations provided by ReSySTER with respect to the experts' recommendations. 
4.2. Sample. The sample of subjects was composed of 30 engineers involved in Scrum projects as well as information about their personal profile and professional competences. These data will be used as input for ReSySTER, allowing the configuration of different working teams. The sample was composed of 30 engineers, 11 women and 19 men, with the average age of 31.2 .

The sample of experts was composed of 4 experts in this scope, 3 men and 1 woman, all of them with considerable experience in Scrum projects, and they were recruited by the authors from among personal contacts. The average age was 33.7. These experts will define a set of WP in a function of real projects and will find the more suitable teams for each one. The results obtained by ReSySTER and the experts will be compared in order to measure the performance of the proposed framework.

4.3. Results. Once the information about competences and the personal profile was defined using the methods explained in Section 3, a set of experts studied the obtained values and made their own team configuration for the WPs based on the requirements previously established using the Delphi method. After that, the results were compared with the output of ReSySTER using different methods and standards.

The Delphi method consists in a separate analysis of the problem made by all the experts involved in the evaluation. Each of them provides a solution and, after that, all the experts have to reach a consensus and provide a final solution for the presented problem. Delphi was developed many years ago, but it still continues to be used and represents a valid instrument for forecasting and supporting decision-making (Landeta, 2006).

For this study, a set of experts were selected to perform the Delphi study. To achieve this, first, Kendall's coefficient of concordance (W or Kendall's W) was computed to measure the level of agreement among the experts. The four experts obtained a coefficient of agreement of $W=0.7121$. Finally, agreement between experts was achieved by applying the Delphi method. Each expert was asked to provide a team configuration for a WP having all the information about people's competences and personal profile. After that, the experts discussed their opinions and configurations to provide a final and joint decision of the best team configuration in each case. These final decisions were compared with the ones provided by ReSySTER.

4.3.1. Phase 1, Test 1: Best team. This test found the best general team for a specific WP selected from the set of 10 real WPs. In this case the best team is selected regardless of the competences defined for the WP and only taking into account the personal profile and professional competences of the SAP set.

In order to determine the accuracy of the ReSySTER results, Precision and Recall and F1 measures were used to measure the degree of relevance of the recommendations provided by the system with respect to the experts' recommendation. The following results describe several perspectives regarding the implementation of this analysis. The results of this first test were analyzed in two steps.

In the first step the configuration teams recommended by the experts were considered correct recommendations, divided into three analyses. First, the overall result establishes that, if the first or second recommendation of ReSySTER coincides with those recommended in the same order by the experts, it is then considered a correct team. Next, the second analysis determines that, if the first team recommended by ReSySTER coincides with the first recommendation of the experts, it is then considered a correct recommendation. Finally, the last analysis establishes that if the second team recommended by ReSySTER coincides with the second recommendation of the experts it is then considered a correct team. Table 8 shows the values obtained in the first Precision and Recall and F1 study. Precision, Recall and F1 values are the same because the number of correct recommendations is the same as the number of recommendations offered by ReSySTER

For the second step, each recommendation is considered correct when ReSySTER and the experts provided the same order for the two teams. Table 10 shows Precision and Recall results for each ReSySTER recommendation with respect to the two teams offered by the expert. In this particular case, it is important to note that Precision and Recall scores are different, since correct teams are not equal to suggested teams.

4.3.2. Phase 1, Test 2: Team with a minor gap. This test found the team with a minor gap for each WP selected from the set of ten real WPs. In this case ReSySTER locates the team that fits better with the competences desired for a specific WP. Moreover, in this test the results were analyzed in two steps. In the first step the configuration teams recommended by the experts were considered correct recommendations, divided into three analyses: overall, first and second recommendations. Table 8 shows the values obtained in the first Precision and Recall and F1 study. Precision, Recall and F1 values are the same because the number of correct recommendations is the same as the number of recommendations offered by ReSySTER.

For the second analysis, each recommendation is considered correct when ReSySTER and the experts provided the same order for the two teams. Table 10 shows Precision and Recall results for each ReSySTER 
Table 6. Precision and Recall analysis for team recommendations.

\begin{tabular}{|c|c|c|c|}
\hline & Precision & Recall & F1 \\
\hline \hline Overall ReSySTER recommendations vs. overall expert recommendations & 0.517 & 0.517 & 0.517 \\
First ReSySTER recommendation vs. first expert recommendation & 0.567 & 0.567 & 0.567 \\
Second ReSySTER recommendation vs. second expert recommendation & 0.467 & 0.467 & 0.467 \\
\hline
\end{tabular}

Table 7. Precision and Recall values for both expert recommendations.

\begin{tabular}{|c|c|c|c|}
\hline & Precision & Recall & F1 \\
\hline \hline Both ReSySTER Recommendations vs. both expert recommendations & 0.667 & 0.333 & 0.444 \\
\hline
\end{tabular}

recommendation with respect to the two teams offered by the expert. In this particular case, it is important to note that Precision and Recall scores are different, since correct teams are not equal to suggested teams.

4.3.3. Phase 2, Test 1: Ranked teams. The test of Phase 2 was oriented towards the assignment of the best team to a set of 10 real WPs. This experiment was more complex, because all teams should be evaluated for all WPs in order to assure the best fit in each case. To this end, ReSySTER established a ranking of teams for each WP. Therefore, taking into account that a team could be assigned only to one WP, it is possible that the same team was the best for several WPs. In this situation, ReSySTER iterates in order to solve these conflicts by assigning a team to the WP with the highest evaluation. In the case of several teams with the maximum evaluation, the selected team is the one with the lowest evaluation for the rest of WPs, allowing the assignment of other good teams for other WPs. The iteration ends when all teams are assigned and no conflicts have appeared or after two iterations without changes (because of conflicts). In the case of conflicts, the project leader is responsible for the final assignment.

The evaluation in this phase was based on the assignment of the team members to the 10 WPs. By means of the Delphi method, experts proposed a team assignment for each of the WP. In this case, only a precision measure has sense because the employed measure is the percentage of similarity between ReSySTER assignments and expert assignments.

4.4. Discussion. Considering the results presented in the previous subsections, we found that the precision of ReSySTER proposing the best team for a WP is $51.7 \%$ with respect the expert recommendation. This is a good result, taking into account the subjectivity of the expert decisions. If the order of the recommendations is not considered, the precision of ReSySTER increases up to $66.7 \%$. It is important to note that a correct result has been considered only if the team proposed by ReSySTER is the same as that proposed by experts. The results of
Test 2 show that ReSySTER approaches the teams with fewer gaps with a precision of $56.7 \%$ with respect to the expert recommendations. Once again the precision reflects the subjectivity of this kind of decisions: the gap calculated by experts is based on their experience and achieving a high degree of approximation is quite difficult. In this second experiment, omitting the order of the expert recommendation, we found that the precision increases up to $63.3 \%$, in a similar way as in Experiment 1. Finally, the experiment (phase 2, test 1) yields a precision of $45.9 \%$. In this latter case, which considers the assignment of the SAP to all WPs, the subjectivity is added to the fact that the error of one team member in one WP implies at least one additional error in another WP in which one incorrect SAP has been assigned. Taking into account the opinion of the experts about the obtained results, they can be considered good.

In order to corroborate the opinion of the experts, the obtained results were compared with those of other recommender systems. Table 11 shows the precision results of three recommender systems developed by the authors. Although the domains of these recommender systems are heterogeneous, they share the subjectivity of the expert decisions (Garcia-Crespo et al., 2011a; 2011b) and the classification (Garcia-Crespo et al., 2010). Sem-fit is a recommender system for the touristic information. As shown, the obtained precision value was $58 \%$, which is comparable with the result obtained by ReSySTER. SINVLIO was developed for the recommendation of investment portfolios. The obtained precision value was $32 \%$, which is clearly inferior to that obtained by ReSySTER. Finally, SEMO is a framework for customer social networks analysis based on emotions. The precision of this system in the best scenario is $78 \%$ higher than the percentage achieved by ReSySTER. With respect to SINVLIO and Sem-Fit, the results of ReSySTER are promising, similar to the best of the values obtained by these systems. With respect to SEMO, we found that the performance of ReSySTER is worse than other two approaches. This can be explained by the fact that SEMO classifies emotions clearly defined at the moment of the evaluation. It makes fine tuning of the system easier, because the results to obtain are not dependent 
Table 8. Precision and Recall analysis for team with minor gap recommendations.

\begin{tabular}{|c|c|c|c|}
\hline & Precision & Recall & F1 \\
\hline \hline Overall ReSySTER recommendations vs. overall expert recommendations & 0.567 & 0.567 & 0.567 \\
First ReSySTER recommendation vs. first expert recommendation & 0.600 & 0.600 & 0.600 \\
Second ReSySTER recommendation vs. second expert recommendation & 0.533 & 0.533 & 0.533 \\
\hline
\end{tabular}

Table 9. Precision and Recall values for both expert recommendation teams with a minor gap.

\begin{tabular}{|c|c|c|c|}
\hline & Precision & Recall & F1 \\
\hline \hline Both ReSySTER recommendations vs. both expert recommendations & 0.633 & 0.317 & 0.422 \\
\hline
\end{tabular}

on subjective expert judgements. For this reason we can conclude that the results obtained by ReSySTER are quite acceptable, compared with similar approaches, and that, in future works, the objective will be the improvement of the recommendations in order to achieve or improve the level of SEMO.

\section{Conclusions}

Software development is a human centric and sociotechnical activity influenced by personnel factors. Furthermore, software workers do not work in isolation. Thus, team composition is one of the main issues in project management. In this environment, agile methods, such as Scrum, have been gaining acceptance in the mainstream software development community because in some situations their use increases the probability of successful development of software (Salo and Abrahamsson, 2008).

This article has presented ReSySTER, a hybrid recommender system for Scrum team roles based on fuzzy logic, rough set theory and semantic technologies. ReSysTER gives recommendations for team formation in Scrum driven projects based on hybrid techniques. Hence, it is designed to assist project managers in configuring the best team for the WPs defined by Scrum. This recommendation is based on determining the best team based on the staff available for the project and the competences required for each work package.

The use of ontologies in ReSySTER enables the utilization of shared vocabularies in order to unify the language used by the experts in the domain. In this way, the characterization of WPs and competencies can be performed without ambiguity in the language. Thus, the categorization of personnel and WPs is made by selecting the appropriate terms of the proposed ontologies. Meanwhile, rough set theory allows the extraction of classification rules based on previous assessments. Rough set theory also allows the determination of the relevant attributes for the categorization. By means of this technique, a set of rules has been obtained in order to approximate the competence level of each member of the SAP.
The fuzzy components of RESySTER can work effectively with many parameters and non-uniform variables suggesting that they can deal with most of the drawbacks of more conventional techniques. Inside ReSySTER these fuzzy components are used in two steps of the framework. Firstly, the numerical values (competence level) of the WP competences will be transformed into a linguistic value based on the IPMA level certification. Secondly, the personal profile and the professional competences of the staff with continuous values are transformed into discrete values through a fuzzyfication process based on fuzzy sets. Through this process the potential uncertainty associated with these variables is reduced and the matching between the staff competences and the competences required by the WPs is facilitated.

The results were defined as accurate, with a considerable degree of similarity between ReSysTER and the expert recommendations. Various tests were performed to search for a specific WP, the best team or the team with a minor gap. Besides, in the last test several WPs were taken into account with the aim to obtain ranked teams. Therefore, the results were considered reliable taking into account the subjective aspects of the decision-making of the experts and the degree of similarity between team recommendations.

Further research should be centered on the collaboration between project managers in the characterization of the SAP set and the definition of WPs. Also the inclusion of a new type of recommendations, such us balanced teams, and new metrics such us mean average precision and mean reciprocal rank, could be performed to improve the proposed system.

\section{Acknowledgment}

This work is supported by the Spanish Ministry of Science and Innovation under the project FLORA: Financial Linked Open Data-based Reasoning and Management for Web Science. 
Table 10. Precision value for the ranked team test

\begin{tabular}{|c|c|}
\hline & Precision \\
\hline \hline ReSySTER recommendations vs. expert recommendations & 0.459 \\
\hline
\end{tabular}

Table 11. Comparison of precision values from other systems.

\begin{tabular}{|c|c|}
\hline & Precision \\
\hline \hline ReSySTER & 0.517 \\
\hline SINVLIO (Garcia-Crespo et al., 2011b) & 0.320 \\
\hline SEMFIT (Garcia-Crespo et al., 2011a) & 0.580 \\
\hline SEMO (Garcia-Crespo et al., 2010) & 0.780 \\
\hline
\end{tabular}

\section{References}

Acuña, S. and Juristo, N. (2004). Assigning people to roles in software projects, Software Practice and Experience 34(7): 675-696.

Acuña, S., Juristo, N. and Moreno, A. (2006). Emphasizing human capabilities in software development, IEEE Software 23(2): 94-101.

Barcus, A. and Montibeller, G. (2008). Supporting the allocation of software development work in distributed teams with multi-criteria decision analysis, Omega 36(3): 464-475.

Barreto, A., Barros, M. and Werner, C. (2008). Staffing a software project: A constraint satisfaction and optimization-based approach, Computers \& Operations Research 35(10): 3073- 3089.

Beck, K. (2001). Extreme Programming Explained: Embrace Change, Addison-Wesley, Reading, MA.

Benjamin, C., Beyer, M., Blampied, N., Duncan, B., Fenster, K., Fitzgerald, D., Goff, S., Henrie, M., Hubbard, B., Ireland, L., Johnston, R., Lindsay, C., Waller, R., Wideman, M. and Youker, R. (2008). USA National Competence Baseline, American Society for the Advancement of Project Management, Colorado Springs, $\mathrm{CO}$.

Casado-Lumbreras, C., Colomo-Palacios, R., Soto-Acosta, P. and Misra, S. (2011). Culture dimensions in software development industry: The effects of mentoring, Scientific Research and Essays 6(11): 2403-2412.

Caupin, G., Knoepfel, H., Koch, G., Pannenbacker, K., Perez-Polo, F. and Seabury, C. (2006). IPMA Competence Baseline: ICB, Version 3.0, International Project Management Association, Nijkerk.

Celik, M., Er, I. and Topcu, Y. (2009). Computer-based systematic execution model on human resources management in maritime transportation industry: The case of master selection for embarking on board merchant ships, Expert Systems with Applications 36(2/1): 10481060.

Chang, C., Christensen, M. and Zhang, T. (2001). Genetic algorithms for project management, Annals of Software Engineering 11(1): 107-139.

Chi, Y. and Chen, C. (2009). Project teaming: Knowledge-intensive design for composing team members, Expert Systems with Applications 36(5): 9479-9487.
Cockburn, A. (2000). Writing Effective Use Cases, The Crystal Collection for Software Professionals, Addison Wesley, Reading, MA.

Colomo-Palacios, R., Casado-Lumbreras, C., Soto-Acosta, P. and Garcia-Crespo, A. (2011). Using the affect grid to measure emotions in software requirements engineering, Journal of Universal Computer Science 17(9): 1281-1298.

Colomo-Palacios, R., Tovar-Caro, E., García-Crespo, A. and Gómez-Berbís, J. (2010). Identifying technical competences of it professionals: The case of software engineers, International Journal of Human Capital and Information Technology Professionals 1(1): 31-43.

Do, S.T., Nguyen, T.T., Woo, D.-M. and Park, D.-C. (2010). Standard additive fuzzy system for stock price forecasting, in N.T. Nguyen, M.T. Le and J. Świątek (Eds.), Proceedings of the 2nd International Conference on Intelligent Information and Database Systems, Part II, Springer-Verlag, Berlin/Heidelberg, pp. 279-288.

Fuentes-Fernandez, R., Garcia-Magariño, I., Gómez-Rodríguez, A. and González-Moreno, J. (2010). A technique for defining agent-oriented engineering processes with tool support, Engineering Applications of Artificial Intelligence 23(3): 432-444.

Garcia-Crespo, A., Colomo-Palacios, R., Gomez-Berbis, J. and Mencke, M. (2009). BMR: Benchmarking metrics recommender for personnel issues in software development projects, International Journal of Computational Intelligence Systems 2(3): 257-267.

Garcia-Crespo, A., Colomo-Palacios, R., Gomez-Beris, J. and Ruiz-Mezcua, B. (2010). SEMO: A framework for customer social networks analysis based on semantics, Journal of Information Technology 25(2): 178-188.

Garcia-Crespo, A., Lopez-Cuadrado, J., Colomo-Palacios, R., Gonzalez-Carrasco, I. and Ruiz-Mezcua, B. (2011a). Sem-fit: A semantic based expert system to provide recommendations in the tourism domain, Expert Systems with Applications 38(10): 13310-13319.

Garcia-Crespo, A., Lopez-Cuadrado, J., Gonzalez-Carrasco, I., Colomo-Palacios, R. and Ruiz-Mezcua, B. (2011b). SINVLIO: Using semantics and fuzzy logic to provide individual investment portfolio recommendations, Expert Systems with Applications 38(10): 13310-13319.

Gruber, T. (1993). A translation approach to portable ontology specifications, Knowledge Acquisition 5(2): 199-220. 
Hadavandi, E., Shavandi, H. and Ghanbari, A. (2010). Integration of genetic fuzzy systems and artificial neural networks for stock price forecasting, Knowledge-Based Systems 23(8): 800-808.

Highsmith, J. and Orr, K. (2000). Adaptive Software Development: A Collaborative Approach to Managing Complex Systems, Dorset House Publishing, New York, NY.

Imai, S., Lin, C., Watada, J. and Tzeng, G. (2008). Rough sets approach to human resource development of information technology corporations, International Journal of Simulation 9(2): 31-42.

Kluska-Nawarecka, S., Wilk-Ko, D., Regulski, K. and Dobrowolski, G. (2011). Rough sets applied to the roughcast system for steel castings, in N.T. Nguyen, C.-G. Kim and A. Janiak (Eds.), Proceedings of the 3rd International Conference on Intelligent Information and Database Systems, Part II, Springer-Verlag, Berlin/Heidelberg, pp. 52-61.

Landeta, J. (2006). Current validity of the Delphi method in social sciences, Technological Forecasting and Social Change 73(5): 467-482.

Lee, S. and Yong, H. (2010). Distributed agile: Project management in a global environment, Empirical Software Engineering 15(2): 204-217.

Li, C. and Chan, F. (2011). Complex-fuzzy adaptive image restoration: An artificial-bee-colony-based learning approach, in N.T. Nguyen, C.-G. Kim and A. Janiak (Eds.), Proceedings of the 3rd International Conference on Intelligent Information and Database Systems, Part II, Springer-Verlag, Berlin/Heidelberg, pp. 90-99.

Li, C. and Chiang, T.-W. (2011). Function approximation with complex neuro-fuzzy system using complex fuzzy sets: A new approach, New Generation Computing 29(3): 261-276.

Licorish, S., Philpott, A. and MacDonell, S. (2009). Supporting agile team composition: A prototype tool for identifying personality (in) compatibilities, Proceedings of the 2009 ICSE Workshop on Cooperative and Human Aspects on Software Engineering, Vancouver, Canada, pp. 66-73.

Liu, J., Wang, W., Golnaraghi, F. and Kubica, E. (2010). A neural fuzzy framework for system mapping applications, Knowledge-Based Systems 23(6): 572-579.

Mahmoud, T.A. (2011). Adaptive control scheme based on the least squares support vector machine network, International Journal of Applied Mathematics and Computer Science 21(4): 685-696, DOI: 10.2478/v10006-011-0054-6.

Malinowski, J., Weitzel, T. and Keim, T. (2008). Decision support for team staffing: An automated relational recommendation approach, Decision Support Systems 45(3): 429- 447.

Malyszko, D. and Stepaniuk, J. (2011). Subspace entropy maps for rough extended framework, in N.T. Nguyen, C.-G. Kim and A. Janiak (Eds.), Proceedings of the 3rd International Conference on Intelligent Information and Database Systems, Part II, Springer-Verlag, Berlin/Heidelberg, pp. 42-51.
Moe, N., Dingsoyr, T. and Dyba, T. (2010). A teamwork model for understanding an agile team: A case study of a Scrum project, Information and Software Technology 52(5): 480-491.

Mohanty, R., Ravi, V. and Patra, M. (2010). The application of intelligent and soft-computing techniques to software engineering problems: A review, International Journal of Information and Decision Sciences 2(3): 233-272.

Naveh, Y., Richter, Y., Altshuler, Y., Gresh, D. and Connors, D. (2007). Workforce optimization: Identification and assignment of professional workers using constraint programming, IBM Journal of Research and Development 51(3/4): 263-279.

Negnevitsky, M. (2005). Artificial Intelligence: A Guide to Intelligent Systems, Addison-Wesley Longman, Boston, MA.

Nerur, S. and Balijepally, V. (2007). Theoretical reflections on agile development methodologies, Communications of the ACM 50(3): 79-83.

Nowicki, R.K. (2010). On classification with missing data using rough-neuro-fuzzy systems, International Journal of Applied Mathematics and Computer Science 20(1): 55-67, DOI: $10.2478 / v 10006-010-0004-8$.

Palmer, S. and Felsing, M. (2002). A Practical Guide to Feature Driven Development, Prentice Hall, Upper Saddle River, NJ.

Pawlak, Z. (1982). Rough sets, International Journal of Parallel Programming 11(6): 341-356.

Pino, F., Pedreira, O., García, F., Luaces, M. and Piattini, M. (2010). Using Scrum to guide the execution of software process improvement in small organizations, Journal of Systems and Software 83(10): 1662- 1677.

Qin, H., Ma, X., Herawan, T. and Zain, J. M. (2011). An adjustable approach to interval-valued intuitionistic fuzzy soft sets based decision making, in N.T. Nguyen, C.-G. Kim and A. Janiak (Eds.), Proceedings of the 3rd International Conference on Intelligent Information and Database Systems, Part II, Springer-Verlag, Berlin/Heidelberg, pp. 80-89.

Salo, O. and Abrahamsson, P. (2008). Agile methods in European embedded software development organisations: A survey on the actual use and usefulness of extreme programming and Scrum, IET Software 2(1): 58-64.

Schwaber, K. and Beedle, M. (2002). Agile Software Development with Scrum, Pearson Prentice-Hall, Upper Saddle River, NJ.

Strnad, D. and Guid, N. (2010). A fuzzy-genetic decision support system for project team formation, Applied Soft Computing 10(4): 1178-1187.

Takeuchi, H. and Nonaka, I. (1986). The new new product development game, Harvard Business Review 64(1): 137-146.

Toroslu, I. and Arslanoglu, Y. (2007). Genetic algorithm for the personnel assignment problem with multiple objectives, Information Sciences 177(3): 787-803. 
Valencia-Garcia, R., Garcia-Sanchez, F., Castellanos-Nieves, D., Fernandez-Breis, J. and Toval, A. (2010). Exploitation of social semantic technology for software development team configuration, IET Software 4(6): 373-385.

Vlaanderen, K., Jansen, S., Brinkkemper, S. and Jaspers, E. (2011). The agile requirements refinery: Applying Scrum principles to software product management, Information and Software Technology 53(1): 58-70.

Wang, J. and Lin, Y.-I. (2003). A fuzzy multicriteria group decision making approach to select configuration items for software development, Fuzzy Sets and Systems 134(3): 343-363.

West, D., Grant, T., Gerush, M. and D'Silva, D. (2010). Agile development: Mainstream adoption has changed agility, Forrester Research, (07/10/2011), http://www. forrester.com/.

Wi, H., Oh, S., Mun, J. and Jung, M. (2009). A team formation model based on knowledge and collaboration, Expert Systems with Applications 36(5): 9121-9134.

Xie, G., Xiong, R. and Church, I. (1998). Comparison of kinetics, neural network and fuzzy logic in modelling texture changes of dry peas in long time cooking, LLWTFood Science and Technology 31(7-8): 639-647.

Zadeh, L. (1965). Application of fuzzy set theory, Fuzzy Sets, Information and Control 8(3): 338-353.

Zhong, N. and Skowron, A. (2001). A rough set-based knowledge discovery process, International Journal of Applied Mathematics and Computer Science 11(1): 603-619.

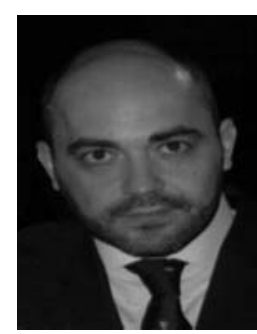

Ricardo Colomo-Palacios is an associate professor at the Computer Science Department of the Carlos III University of Madrid. His research interests include applied research in information systems, software project management, people in software projects, and social and Semantic Web. He received his Ph.D. in computer science from the Technical University of Madrid (2005). He also holds an M.B.A. from the IE (2002). He has been working as a software engineer, a project manager and a software engineering consultant in several companies including the Spanish IT leader INDRA. He is also an editorial board member and associate editor for several international journals and conferences, and the editor-in-chief of the International Journal of Human Capital and Information Technology Professionals.

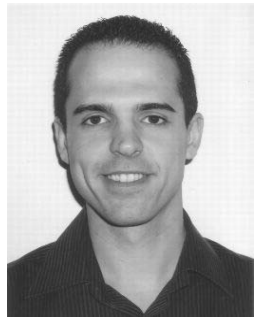

Israel González-Carrasco is an assistant professor in the Computer Science Department of the Carlos III University of Madrid. He holds a $\mathrm{Ph} . \mathrm{D}$. degree in computer science. He is a coauthor of several papers and his main lines of research are neural networks, expert systems and software engineering. He is involved in several international projects and is a member of the editorial reviewer board of the International Journal of Artificial Intelligence, Neural Networks, and Complex Problem-Solving Technologies.

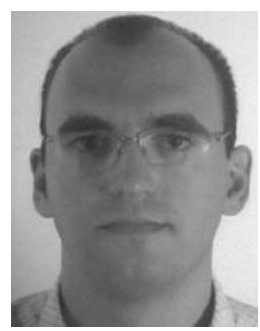

José Luis López-Cuadrado is an assistant professor in the Computer Science Department at the Carlos III University of Madrid. He holds a Ph.D. degree in computer science. His research is focused on web based and collaborative expert systems applications, neural networks, software engineering and processes improvement. Also, he is a co-author of several contributions published in international congress proceedings and journals.

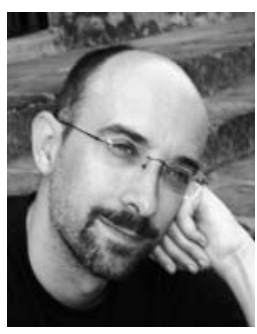

Ángel García-Crespo, doctor of industrial engineering, Technical University of Madrid. Award of the J.A. Artigas Institute for the best Ph.D. thesis. Executive M.B.A. by the IE. Associate professor at the Carlos III University of Madrid. Director of the technological development and innovation promotion institute of Pedro Juan de Lastanosa. Director of the advanced systems integration Softlab research team. Participant in European Union projects, the 4 th and the 5th Marco program, and CICYT and CAM projects, including a significant number of $\mathrm{R}+\mathrm{D}$ projects with companies. Author of more than a hundred articles in congress proceedings as well as Spanish and international journals.

Received: 23 April 2012 